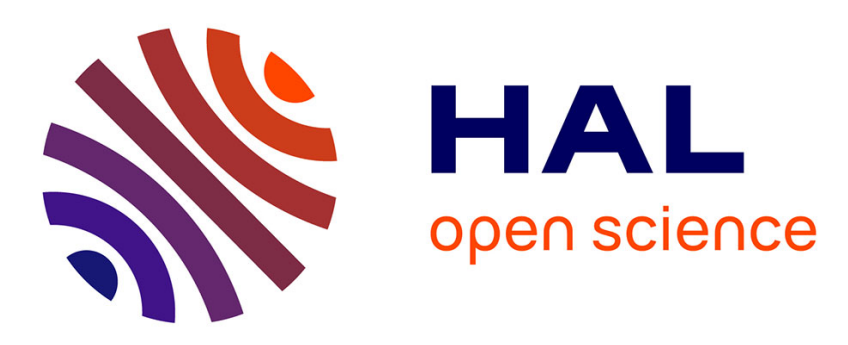

\title{
Apprentissage des propulsions synchrone et asynchrone en fauteuil roulant manuel
}

Ilona Alberca, Marion Combet, Marjolaine Astier, Eric Watelain, Didier Pradon, Arnaud Faupin

\section{- To cite this version:}

Ilona Alberca, Marion Combet, Marjolaine Astier, Eric Watelain, Didier Pradon, et al.. Apprentissage des propulsions synchrone et asynchrone en fauteuil roulant manuel. XIXe Journées d'Etudes Francophones en Activités Physiques Adaptées (AFAPA 2018), May 2018, Toulon, France. 33, pp.S39-S40, 2018, Science \& Sports. 10.1016/j.scispo.2018.03.063 . hal-03328540

HAL Id: hal-03328540

https://hal.science/hal-03328540

Submitted on 30 Aug 2021

HAL is a multi-disciplinary open access archive for the deposit and dissemination of scientific research documents, whether they are published or not. The documents may come from teaching and research institutions in France or abroad, or from public or private research centers.
L'archive ouverte pluridisciplinaire HAL, est destinée au dépôt et à la diffusion de documents scientifiques de niveau recherche, publiés ou non, émanant des établissements d'enseignement et de recherche français ou étrangers, des laboratoires publics ou privés. 
Ilona Alberca ${ }^{1}$, Marion Combet', Marjolaine Astier ${ }^{1}$, Eric Watelain², Didier Pradon ${ }^{3}$ \& Arnaud Faupin ${ }^{1}$

1Université de Toulon, LAMHESS, , EA 6312, 83957 La Garde, France

2Université de Valenciennes, LAMIH UMR UVHC-CNRS 8201, France

3 U1179 End:icap, hôpital Raymond-Poincaré, service de physiologie et d'explorations fonctionnelles, Garches, France

\section{Introduction}

Deux modes de propulsion existent en FRM : synchrone (SYN), les deux bras poussent en même temps et asynchrone (ASY), les bras poussent en alternance. Beaucoup d'études sur l'apprentissage du FRM existent mais peu s'intéressent à la propulsion ASY. Or, la propulsion ASY est pourtant utilisée en handisport ${ }^{123}$.

\section{Objectif : analyser les différences d'apprentissage de ces deux techniques de propulsion}

\section{Méthode}

Deux sprints, en conditions SYN et ASY, avant (T1) et après $12 \mathrm{~h}$ de pratique sportive (T2) incluant ces deux modes de propulsion, ont été réalisés à l'aide une roue instrumentée. Le taux d'augmentation de la force totale (Ror) lors de la saisie de la main courante, la cadence, la force totale, la vitesse et puissance maximale ont été mesurées. Statistique : test non paramétrique de Wilcoxon pour échantillons appariés.

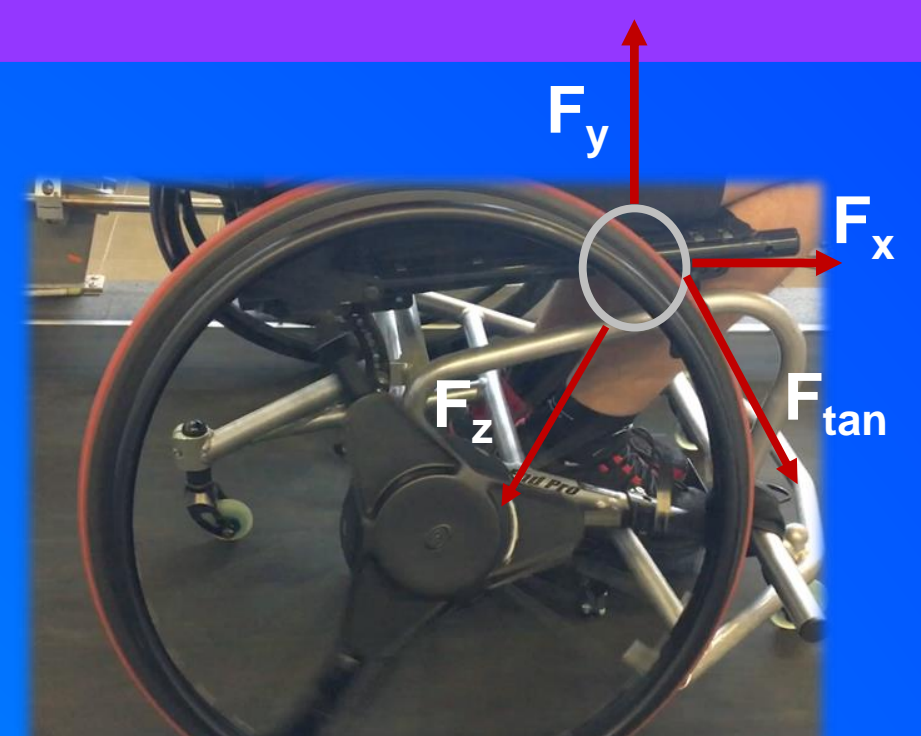

\section{Résultats}

\begin{tabular}{|c|c|c|c|c|c|}
\hline & $\begin{array}{l}\text { Cadence } \\
\text { (coût.min-1) }\end{array}$ & $\begin{array}{l}\text { Force totale } \\
\text { (N) }\end{array}$ & $\begin{array}{l}\text { Ror } \\
\left(\mathrm{N} . \mathrm{s}^{-1}\right)\end{array}$ & $\begin{array}{c}\text { Vitesse } \\
\text { maximale } \\
\left(\mathrm{m} \cdot \mathrm{s}^{-1}\right)\end{array}$ & $\begin{array}{l}\text { Puissance } \\
\text { (W) }\end{array}$ \\
\hline ASY T1 & $\begin{array}{c}100,56 \\
( \pm 25,24)\end{array}$ & $\begin{array}{c}108,69 \\
( \pm 41,39)\end{array}$ & $\begin{array}{c}1117,15 \\
( \pm 486,62)\end{array}$ & $\begin{array}{c}2,68 \\
( \pm 0,37)\end{array}$ & $\begin{array}{c}183,96 \\
( \pm 98,40)\end{array}$ \\
\hline ASY T2 & $\begin{array}{c}100,52 \\
( \pm 22,88)\end{array}$ & $\begin{array}{c}132,56 \\
( \pm 41,38)\end{array}$ & $\begin{array}{c}1491,70 * \\
( \pm 561,89)\end{array}$ & $\begin{array}{l}3,20 * * \\
( \pm 0,61)\end{array}$ & $\begin{array}{c}221,54^{*} \\
( \pm 102,28)\end{array}$ \\
\hline SYN T1 & $\begin{array}{c}106,96 \\
( \pm 21,25)\end{array}$ & $\begin{array}{c}123,30 \\
( \pm 44,24)\end{array}$ & $\begin{array}{c}1261,98 \\
( \pm 566,81)\end{array}$ & $\begin{array}{c}3,04 \\
( \pm 0,46)\end{array}$ & $\begin{array}{c}208,41 \\
( \pm 107,63)\end{array}$ \\
\hline SYN T2 & $\begin{array}{c}112,20 \\
( \pm 22,76)\end{array}$ & $\begin{array}{l}147,36 * \\
( \pm 47,03)\end{array}$ & $\begin{array}{l}1641,08^{*} \\
( \pm 777,17)\end{array}$ & $\begin{array}{c}3,60^{*} \\
( \pm 0,55)\end{array}$ & $\begin{array}{c}268,78^{*} \\
( \pm 135,98)\end{array}$ \\
\hline
\end{tabular}

\section{Discussion}

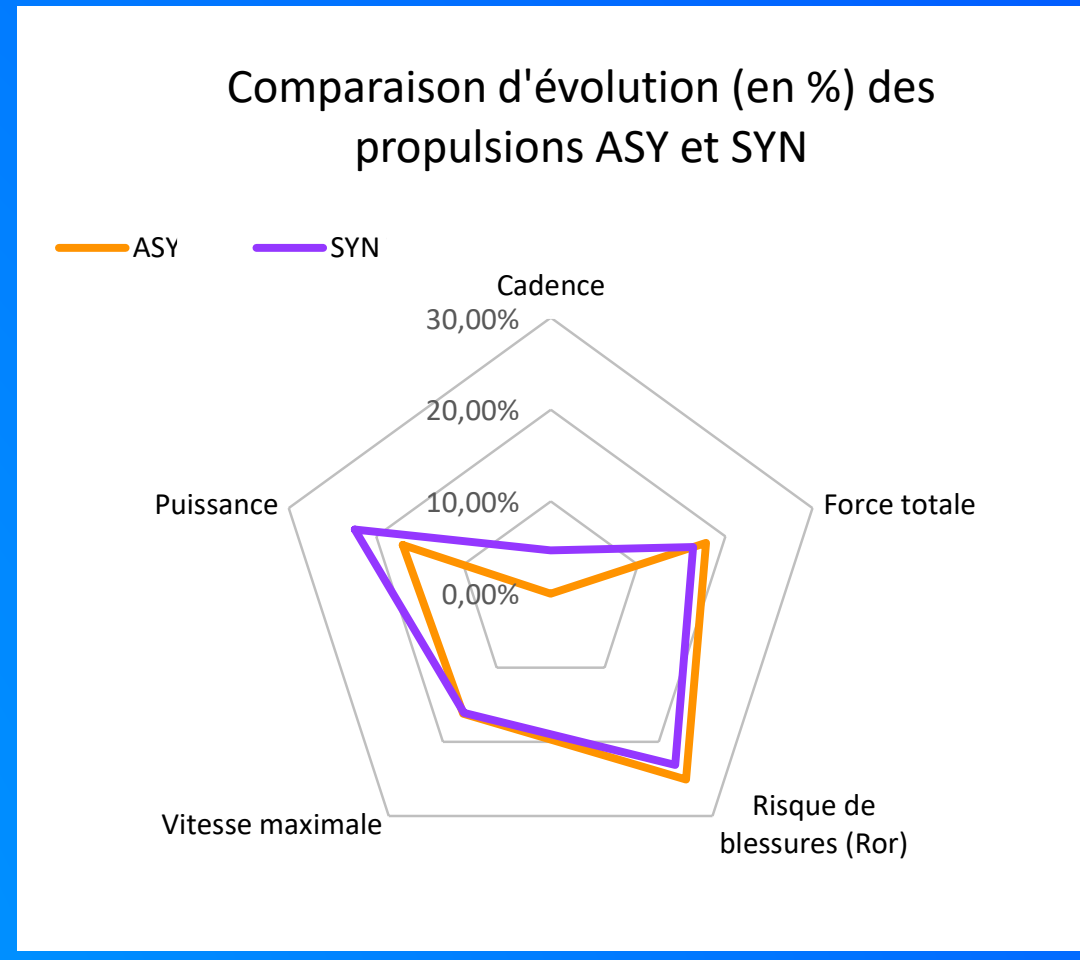

Les pourcentages d'évolution entre propulsions SYN et ASY restent très proches avec une amélioration des paramètres de performance sans modification de la cadence. L'augmentation du Ror pouvant s'expliquer par l'amélioration de la vitesse maximale. Nous pouvons préconiser à partir des ces premiers résultats des phases d'apprentissages spécifilques en mode ASYN chez les handisportifs. 